\title{
Controllability of partial differential equations
}

\author{
Yacine Chitour, Emmanuel Trélat ${ }^{\dagger}$
}

\section{Contents}

1 Semigroup theory, and Cauchy problems in Banach spaces 2

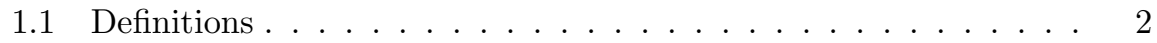

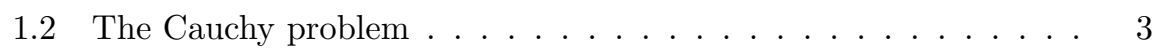

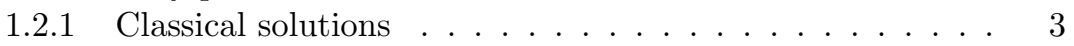

1.2 .2 Weak solutions . . . . . . . . . . . . . . 4

1.3 The nonhomogeneous initial value problem . . . . . . . . . 7

2 Controllability and observability in Banach spaces 8

2.1 A short overview on controllability of finite dimensional linear control systems . . . . . . . . . . . . . . . . . 8

2.2 Controllability of linear partial differential equations in Banach spaces ......................... 8

2.2.1 Admissible control and observation operators . . . . . . 9

2.2.2 Necessary and sufficient conditions for controllability in

Banach spaces . . . . . . . . . . . . . 14

3 Semidiscrete approximations of infinite dimensional linear control systems in Hilbert spaces $\quad 17$

3.1 Introduction . . . . . . . . . . . . . . . . . . 17

3.2 Uniform controllability of semidiscrete approximations of parabolic control systems . . . . . . . . . . . . . . . . . . . 19

\footnotetext{
*Laboratoire des signaux et systèmes, Supelec, Plateau du Moulon, Université Paris-Sud

†Université Paris-Sud, Laboratoire ANEDP, Mathématique, UMR 8628, Bât. 425, 91405 Orsay cedex, France (Yacine.Chitour@lss.supelec.fr, Emmanuel.Trelat@math.u-psud.fr).
} 


\section{Semigroup theory, and Cauchy problems in Banach spaces}

In this section, we recall some basic elements of semigroup theory (see [25]).

\subsection{Definitions}

Let $X$ be a Banach space.

Definition 1.1. A one-parameter family $(S(t))_{t \geq 0}$ of bounded linear operators from $X$ into $X$ is called a semigroup of bounded linear operators on $X$ if

- $S(0)=I$,

- $S(t+s)=S(t) S(s)$, for all $t, s \geq 0$.

The linear operator $A: D(A) \rightarrow X$, defined on the domain

$$
D(A)=\left\{y \in X \mid \lim _{\substack{t \rightarrow 0 \\ t>0}} \frac{S(t) y-y}{t} \text { exists }\right\},
$$

by

$$
A y=\lim _{\substack{t \rightarrow 0 \\ t>0}} \frac{S(t) y-y}{t},
$$

for $y \in D(A)$, is called the infinitesimal generator of the semigroup $S(t)$.

Definition 1.2. A semigroup $S(t)$ of bounded linear operators is said

- uniformly continuous if

$$
\lim _{\substack{t \rightarrow 0 \\ t>0}}\|S(t)-I\|=0
$$

- strongly continuous (or $C_{0}$ semigroup) if

$$
\lim _{\substack{t \rightarrow 0 \\ t>0}} S(t) y=y
$$

for every $y \in X$.

Theorem 1.1. A linear operator $A$ is the infinitesimal generator of a uniformly continuous semigroup if and only if $A$ is bounded.

In what follows, $\rho(A)$ denotes the resolvent set of $A$, that is, the set of complex numbers $\lambda$ such that $\lambda I-A$ is boundedly invertible. For $\lambda \in \rho(A)$, let

$$
R(\lambda, A)=(\lambda I-A)^{-1}
$$

denote the resolvent of $A$. 
Theorem 1.2. Let $S(t)$ be a $C_{0}$ semigroup. There exist constants $\omega \geq 0$ and $M \geq 1$ such that

$$
\|S(t)\| \leq M \mathrm{e}^{\omega t},
$$

for every $t \geq 0$.

$A$ linear operator $A$ is the infinitesimal generator of $S(t)$ if and only if

(i) $A$ is closed, and $D(A)$ is dense in $X$;

(ii) $(\omega,+\infty) \subset \rho(A)$, and

$$
\left\|R(\lambda, A)^{n}\right\| \leq \frac{M}{(\lambda-\omega)^{n}},
$$

for every $\lambda$ having a real part $\Re \lambda>\omega$, and every $n \in \mathbb{N}^{*}$.

Remark 1.1. Let $S(t)$ be a semigroup satisfying

$$
\|S(t)\| \leq M \mathrm{e}^{\omega t},
$$

for some $\omega \geq 0$ and $M \geq 1$. Then,

$$
\{\lambda \in \mathbb{C} \mid \Re \lambda>\omega\} \subset \rho(A),
$$

and

$$
R(\lambda, A) y=(\lambda I-A)^{-1} y=\int_{0}^{+\infty} \mathrm{e}^{-\lambda t} S(t) y d t,
$$

for every $y \in X$, and every $\lambda$ such that $\Re \lambda>\omega$.

\subsection{The Cauchy problem}

\subsubsection{Classical solutions}

Let $A: D(A) \rightarrow X$ be a linear operator on the Banach space $X$, such that $D(A)$ is dense in $X$. Consider the Cauchy problem

$$
\begin{aligned}
& \dot{y}(t)=A y(t), \quad \text { for } t \geq 0, \\
& y(0)=y_{0} \in D(A) .
\end{aligned}
$$

Theorem 1.3. Suppose that $A$ is the infinitesimal generator of a $C_{0}$ semigroup $S(t)$ on $X$. Then, the Cauchy problem (1) has a unique solution

$$
y(\cdot) \in C^{0}(0, T ; D(A)) \cap C^{1}(0, T ; X),
$$

given by

$$
y(t)=S(t) y_{0},
$$

for every $t \geq 0$. 
Example 1.1. Let $\Omega \subset \mathbb{R}^{n}$ be a bounded open set having a $C^{1}$ boundary. The Cauchy problem

$$
\begin{aligned}
\dot{y} & =\triangle y \quad \text { in } \Omega, \\
y_{\mid \partial \Omega} & =0, \\
y(0) & =y_{0} \in H_{0}^{1}(\Omega),
\end{aligned}
$$

has a unique solution

$$
y(\cdot) \in C^{0}\left(0,+\infty ; H_{0}^{1}(\Omega)\right) \cap C^{1}\left(0,+\infty ; H^{-1}(\Omega)\right) .
$$

Moreover, there exist $M, \omega>0$ such that

$$
\|y(t, \cdot)\|_{L^{2}(\Omega)} \leq M \mathrm{e}^{-\omega t}\left\|y_{0}(\cdot)\right\|_{L^{2}(\Omega)} .
$$

Example 1.2. Let $\Omega \subset \mathbb{R}^{n}$ be a bounded open set having a $C^{1}$ boundary. The Cauchy problem

$$
\begin{aligned}
\ddot{y} & =\triangle y \quad \text { in } \Omega, \\
y_{\mid \partial \Omega} & =0, \\
y(0) & =y_{0} \in H_{0}^{1}(\Omega), \dot{y}(0)=y_{1} \in L^{2}(\Omega),
\end{aligned}
$$

has a unique solution

$$
y(\cdot) \in C^{0}\left(0,+\infty ; H_{0}^{1}(\Omega)\right) \cap C^{1}\left(0,+\infty ; L^{2}(\Omega)\right) \cap C^{1}\left(0,+\infty ; H^{-1}(\Omega)\right) .
$$

Moreover,

$$
\|\dot{y}\|_{H^{-1}(\Omega)}^{2}+\|y\|_{L^{2}(\Omega)}^{2}=\left\|y_{1}\right\|_{H^{-1}(\Omega)}^{2}+\left\|y_{0}\right\|_{L^{2}(\Omega)}^{2} .
$$

Note that, if the boundary of $\Omega$ is of class $C^{2}$, and if

$$
y_{0} \in H^{2}(\Omega) \cap H_{0}^{1}(\Omega) \text {, and } y_{1} \in H_{0}^{1}(\Omega),
$$

then

$$
y(\cdot) \in C^{0}\left(0,+\infty ; H^{2}(\Omega) \cap H_{0}^{1}(\Omega)\right) \cap C^{1}\left(0,+\infty ; H_{0}^{1}(\Omega)\right) \cap C^{1}\left(0,+\infty ; L^{2}(\Omega)\right),
$$

and

$$
\|\dot{y}\|_{L^{2}(\Omega)}^{2}+\|y\|_{H_{0}^{1}(\Omega)}^{2}=\left\|y_{1}\right\|_{L^{2}(\Omega)}^{2}+\left\|y_{0}\right\|_{H_{0}^{1}(\Omega)}^{2} .
$$

If $y_{0} \in X \backslash D(A)$, then, in general, $y(t)=S(t) y_{0} \notin D(A)$, and thus, $y(t)$ is not solution of (1) in the usual sense. Actually, $y(t)$ is solution in a weaker sense.

\subsubsection{Weak solutions}

Let $S(t)$ be a $C_{0}$ semigroup on the Banach space $X$, with generator $A: D(A) \rightarrow$ $X$. Let $\beta \in \rho(A)$ (if $X$ is real, consider a real such number $\beta$ ). 
Definition 1.3. Let $X_{1}$ denote the Banach space $D(A)$, equipped with the norm

$$
\|y\|_{1}=\|(\beta I-A) y\|,
$$

and let $X_{-1}$ denote the completion of $X$ with respect to the norm

$$
\|y\|_{-1}=\left\|(\beta I-A)^{-1} y\right\|=\|R(\beta, A) y\| .
$$

It is not difficult to prove that the norm \|\|$_{1}$ on $X_{1}$ is equivalent to the graph norm $\|y\|_{G}=\|y\|+\|A y\|$. Therefore, from the closed graph theorem,

- $\left(X_{1},\|\|_{1}\right)$ is complete,

- we get an equivalent norm, for any $\beta^{\prime} \in \rho(A)$.

On the other part, the space $X_{-1}$ does not depend on the specific value of $\beta \in \rho(A)$.

Example 1.3. Let $\Omega \subset \mathbb{R}^{n}$ be an open bounded set having a $C^{2}$ boundary. Then, $A=-\triangle: H_{0}^{1}(\Omega) \cap H^{2}(\Omega) \rightarrow L^{2}(\Omega)$ is an isomorphism. Set $X=L^{2}(\Omega)$. Then,

$$
X_{1}=D(A)=H_{0}^{1}(\Omega) \cap H^{2}(\Omega),
$$

and

$$
X_{-1}=\left(H_{0}^{1}(\Omega) \cap H^{2}(\Omega)\right)^{\prime},
$$

where the dual is considered with respect to the pivot space $X=L^{2}(\Omega)$.

Note that the construction can be generalized so as to obtain a scale of Banach spaces $\left(X_{\alpha}\right)_{\alpha \in \mathbb{R}}$.

Definition 1.4. The adjoint operator $A^{*}: D\left(A^{*}\right) \rightarrow X^{\prime}$, of the operator $A$, is defined by

$$
D\left(A^{*}\right)=\left\{x \in X^{\prime} \mid \exists y \in X^{\prime}, \forall z \in D(A) \quad\langle x, A z\rangle X^{\prime}, X=\langle y, z\rangle_{X^{\prime}, X}\right\},
$$

and, if $x \in D\left(A^{*}\right)$, then $y=A^{*} x$.

Note that, since $D(A)$ is dense in $X$, there exists at most one such $y$.

We endow $D\left(A^{*}\right)$ with the graph norm

$$
\|y\|_{1}=\left\|\left(\beta I-A^{*}\right) y\right\|_{X^{\prime}}
$$

where $\beta \in \rho\left(A^{*}\right)=\rho(A)$.

Note that, if $X$ is reflexive, and if $S(t)$ is a $C_{0}$ semigroup on $X$ with generator $A$, then $S(t)^{*}$ is a $C_{0}$ semigroup on $X^{\prime}$ with generator $A^{*}$.

Theorem 1.4. If $X$ is reflexive, then $X_{-1}$ is isomorphic to $D\left(A^{*}\right)^{\prime}$.

Remark 1.2. One has $X_{1} \subset X \subset X_{-1}$, with continuous and dense embeddings. 
Theorem 1.5. The operator $A: D(A) \rightarrow X$ extends to an operator $A_{-1}$ : $D\left(A_{-1}\right)=X \rightarrow X_{-1}$, and the semigroup $S(t)$ on $X$ extends to a semigroup $S_{-1}(t)$ on $X_{-1}$, generated by $A_{-1}$.

Definition 1.5. For every $y_{0} \in X$, the unique solution

$$
y(t)=S(t) y_{0}
$$

of the Cauchy problem

$$
\begin{aligned}
& \dot{y}(t)=A_{-1} y(t), \quad \text { for } t \geq 0, \\
& y(0)=y_{0},
\end{aligned}
$$

in the space

$$
C^{0}(0,+\infty ; X) \cap C^{1}\left(0,+\infty ; X_{-1}\right)
$$

is called a weak solution.

Example 1.4. Let $\Omega \subset \mathbb{R}^{n}$ be a bounded open set having a $C^{2}$ boundary. The Cauchy problem

$$
\begin{aligned}
\dot{y} & =\triangle y \quad \text { in } \Omega, \\
y_{\mid \partial \Omega} & =0, \\
y(0) & =y_{0} \in L^{2}(\Omega),
\end{aligned}
$$

has a unique (weak) solution

$$
y \in C^{0}\left(0,+\infty ; L^{2}(\Omega)\right) \cap C^{1}\left(0,+\infty ;\left(H_{0}^{1}(\Omega) \cap H^{2}(\Omega)\right)^{\prime}\right) .
$$

Moreover, there exist $M, \omega>0$ such that

$$
\|y(t, \cdot)\|_{L^{2}(\Omega)} \leq M \mathrm{e}^{-\omega t}\left\|y_{0}(\cdot)\right\|_{L^{2}(\Omega)} .
$$

Example 1.5. Let $\Omega \subset \mathbb{R}^{n}$ be a bounded open set having a $C^{2}$ boundary. Consider the Cauchy problem

$$
\begin{aligned}
\ddot{y} & =\triangle y \quad \text { in } \Omega, \\
y_{\mid \partial \Omega} & =0, \\
y(0) & =y_{0}, \quad \dot{y}(0)=y_{1} .
\end{aligned}
$$

- If $y_{0} \in H^{-1}(\Omega)$ and $y_{1} \in\left(H_{0}^{1}(\Omega) \cap H^{2}(\Omega)\right)^{\prime}$, then there is a unique solution

$$
y(\cdot) \in C^{0}\left(0,+\infty ; H^{-1}(\Omega)\right) \cap C^{1}\left(0,+\infty ;\left(H_{0}^{1}(\Omega) \cap H^{2}(\Omega)\right)^{\prime}\right) .
$$

- If $y_{0} \in L^{2}(\Omega)$ and $y_{1} \in H^{-1}(\Omega)$, then there is a unique solution

$$
y(\cdot) \in C^{0}\left(0,+\infty ; L^{2}(\Omega)\right) \cap C^{1}\left(0,+\infty ; H^{-1}(\Omega)\right) .
$$




\subsection{The nonhomogeneous initial value problem}

Consider the Cauchy problem

$$
\begin{aligned}
& \dot{y}(t)=A y(t)+f(t), \quad \text { for } t \geq 0, \\
& y(0)=y_{0},
\end{aligned}
$$

where $A: D(A) \rightarrow X$ generates a $C_{0}$ semigroup $S(t)$ on $X$.

Theorem 1.6. If $y_{0} \in D(A)$, and $f \in L^{1}(0, T ; D(A))$, then (2) admits a unique solution

$$
y \in C^{0}(0, T ; D(A)) \cap C^{1}(0, T ; X),
$$

given by

$$
y(t)=S(t) y_{0}+\int_{0}^{t} S(t-s) f(s) d s .
$$

Note that, if $f \in L^{1}(0, T ; X)$, (3) still makes sense.

Definition 1.6. - If $y_{0} \in X$ and $f \in L^{1}(0, T ; X)$, then $y$ defined by (3) is called mild solution of (2).

- If $y_{0} \in D(A)$ and $f \in C^{0}(0, T ; X)$, and if

$$
y \in C^{0}(0, T ; D(A)) \cap C^{1}(0, T ; X),
$$

then $y$ defined by (3) is called strong solution of (2).

- Assume $X$ reflexive. If $y_{0} \in X_{-1} \simeq D\left(A^{*}\right)^{\prime}$, and $f \in L^{1}\left(0, T ; X_{-1}\right)$, then $y$ defined by

$$
y(t)=S_{-1}(t) y_{0}+\int_{0}^{t} S_{-1}(t-s) f(s) d s,
$$

is called weak solution of (2).

Remark 1.3. The condition $f \in C^{0}(0, T ; X)$ does not ensure the existence of strong solutions. However, we have the following result.

Theorem 1.7. If $y_{0} \in D(A)$ and $f \in C^{1}(0, T ; X)$, then (2) has a unique strong solution.

Corollary 1.8. If $y_{0} \in X$ and $f \in C^{1}\left(0, T ; X_{-1}\right)$ (or $f \in W^{1,1}\left(0, T ; X_{-1}\right)$ ), then (2) has a unique weak solution, such that

$$
y \in C^{0}(0, T ; X) \cap C^{1}\left(0, T ; X_{-1}\right) .
$$




\section{Controllability and observability in Banach spaces}

\subsection{A short overview on controllability of finite dimen- sional linear control systems}

We start the section by recalling some well known results in the finite dimensional context.

Let $T>0$ be fixed. Consider the linear control system

$$
\dot{x}(t)=A x(t)+B u(t),
$$

where $x(t) \in \mathbb{R}^{n}, A$ is a $(n \times n)$-matrix, $B$ is a $(n \times m)$-matrix, with real coefficients, and $u(\cdot) \in L^{2}\left(0, T ; \mathbb{R}^{m}\right)$.

Let $x_{0} \in \mathbb{R}^{n}$. The system (4) is said to be controllable from $x_{0}$ in time $T$ if and only if, for every $x_{1} \in \mathbb{R}^{n}$, there exists $u(\cdot) \in L^{2}\left(0, T ; \mathbb{R}^{m}\right)$ so that the solution $x(\cdot)$ of $(4)$, with $x(0)=x_{0}$, associated with the control $u(\cdot)$, satisfies $x(T)=x_{1}$.

It is well known that the system (4) is controllable in time $T$ if and only if the matrix

$$
\int_{0}^{T} \mathrm{e}^{(T-t) A} B B^{*} \mathrm{e}^{(T-t) A^{*}} d t
$$

called Gramian of the system, is nonsingular (here, $M^{*}$ denotes the transpose of the matrix $M)$. Since we are in finite dimension, this is equivalent to the existence of $\alpha>0$ so that

$$
\int_{0}^{T}\left\|B^{*} \mathrm{e}^{(T-t) A^{*}} \psi\right\|^{2} d t \geq \alpha\|\psi\|^{2},
$$

for every $\psi \in \mathbb{R}^{n}$ (observability inequality).

It is also well known that, if such a linear system is controllable from $x_{0}$ in time $T>0$, then it is controllable in time $T^{\prime}$, for every $T^{\prime}>0$, and from every initial state $x_{0}^{\prime} \in \mathbb{R}^{n}$. Indeed, another necessary and sufficient condition for controllability is the Kalman condition

$$
\operatorname{rank}\left(B, A B, \ldots, A^{n-1} B\right)=n,
$$

wich is independent on $x_{0}$ and $T$.

\subsection{Controllability of linear partial differential equations in Banach spaces}

In this section we review some known facts on controllability of infinite dimensional linear control systems in Banach spaces (see [34, 35, 31]).

The notation $L(E, F)$ stands for the set of linear continuous mappings from $E$ to $F$, where $E$ and $F$ are Banach spaces.

We deal with the infinite dimensional linear control system

$$
\begin{aligned}
& \dot{y}(t)=A y(t)+B u(t), \\
& y(0)=y_{0},
\end{aligned}
$$


where the state $y(t)$ belongs to a Banach space $X$, the control $u(t)$ belongs to a Banach space $U, A: D(A) \rightarrow X$ is the generator of a $C_{0} \operatorname{semigroup} S(t)$ on $X$, and $B \in L\left(U, X_{-1}\right)$.

\subsubsection{Admissible control and observation operators}

The control operator $B$ is said to be bounded if $B \in L(U, X)$, and is called unbounded otherwise (note however that $B$ is a bounded operator from $U$ in $\left.X_{-1}\right)$. Unbounded operators appear naturally when dealing with boundary or pointwise control systems.

A priori, (7) makes sense in $X_{-1}$, and if $u \in L^{2}(0, T ; U)$, then

$$
y(t)=S(t) y_{0}+L_{t} u
$$

where

$$
L_{t} u=\int_{0}^{t} S(t-s) B u(s) d s,
$$

is a weak solution (from now on, $S_{-1}(t)$ is denoted $S(t)$, for the sake of simplicity). Moreover,

$$
L_{t} u \in X_{-1}
$$

and thus

$$
y \in H^{1}\left(0, T ; X_{-1}\right) .
$$

The objective is to characterize control operators $B$ such that $y(t) \in X$, for every $t \geq 0$, whenever $y_{0} \in X$. Note that, if $y_{0} \in X$, then $S(t) y_{0} \in X$, for every $t \geq 0$.

Definition 2.1. $B \in L\left(U, X_{-1}\right)$ is called admissible control operator for $S(t)$ if the weak solution of $(7)$, with $y_{0} \in X$, belongs to $X$, whenever $u \in L^{2}(0, T ; U)$. This is equivalent to requiring

$$
L_{T} \in L\left(L^{2}(0, T ; U), X\right) .
$$

Note that, if $B$ is admissible, then

$$
y \in H^{1}(0, T ; X),
$$

and

$$
\dot{y}=A y+B u \quad \text { in } X_{-1},
$$

almost everywhere on $[0, T]$.

Note also that, in the term $L_{t} u$, the integration is done in $X_{-1}$, but the result is in $X$ whenever $B$ is admissible.

Definition 2.2. Let $Y$ denote a Banach space. Let $S(t)$ be a $C_{0}$ semigroup on $X$, with generator $A$, and let $C \in L(D(A), Y)$. The operator $C$ is called admissible observation operator for $S(t)$ if, for every $T>0$, there exists $C_{T}>0$ such that

$$
\int_{0}^{T}\|C S(t) y\|_{Y}^{2} d t \leq C_{T}\|y\|_{X}^{2}
$$

for every $y \in D(A)$. 
A priori, (8) makes sense for $y \in D(A)$. For $y \in X$, one has to replace $C$ with its $\Lambda$-extension

$$
C_{\Lambda} z=\lim _{\lambda \rightarrow+\infty} C \lambda(\lambda I-A)^{-1} z
$$

also called Lebesgue extension (introduced in [34]). Then, replacing $C$ with $C_{\Lambda}$, (8) makes sense, for every $y \in X$.

Theorem 2.1. Assume that $X$ and $U$ are reflexive, and that $A: D(A) \rightarrow X$ is the generator of a $C_{0}$ semigroup $S(t)$ on $X$. Then, $B \in L\left(U, X_{-1}\right)$ is an admissible control operator for $S(t)$ if and only if $B^{*} \in L\left(D\left(A^{*}\right), U^{\prime}\right)$ is an admissible observation operator for $S(t)^{*}$.

Moreover, the adjoint $L_{T}^{*}$ of $L_{T}$ is given by

$$
\begin{aligned}
\forall y \in D\left(A^{*}\right) & \left(L_{T}^{*} x\right)(t)=B^{*} S(T-t)^{*} x, \quad \forall t \in[0, T] \\
\forall y \in X^{\prime} & \left(L_{T}^{*} x\right)(t)=B_{\Lambda}^{*} S(T-t)^{*} x, \quad \text { for a.e. } t \in[0, T],
\end{aligned}
$$

where, as previously,

$$
B_{\Lambda}^{*} z=\lim _{\lambda \rightarrow+\infty} \lambda B^{*}\left(\lambda I-A^{*}\right)^{-1} z
$$

Note that, for $B$ admissible, $L_{T}: L^{2}(0, T ; U) \rightarrow X$, and $L_{T}^{*}: X^{\prime} \rightarrow$ $L^{2}\left(0, T ; U^{\prime}\right)$.

Example 2.1. Let $\Omega \subset \mathbb{R}^{n}$ be a bounded open set having a $C^{2}$ boundary. Consider the heat equation with boundary Dirichlet control

$$
\begin{aligned}
\dot{y} & =\triangle y \quad \text { in } \Omega, \\
y_{\mid \partial \Omega} & =u(t), \\
y(0) & =y_{0} \in L^{2}(\Omega) .
\end{aligned}
$$

Set $X=L^{2}(\Omega)$, and $A=\triangle: D(A) \rightarrow X$, where

$$
D(A)=X_{1}=H^{2}(\Omega) \cap H_{0}^{1}(\Omega) .
$$

The operator $A$ is selfadjoint, and

$$
X_{-1}=D\left(A^{*}\right)^{\prime}=\left(H^{2}(\Omega) \cap H_{0}^{1}(\Omega)\right)^{\prime},
$$

with respect to the pivot space $L^{2}(\Omega)$. Then,

$$
B^{*} \phi=-\frac{\partial \phi}{\partial \nu} \mid \partial \Omega
$$

for every $\phi \in D\left(A^{*}\right)$, and $B$ is defined by transposition

$$
\langle B u, \phi\rangle_{\left(H^{2}(\Omega) \cap H_{0}^{1}(\Omega)\right)^{\prime}, H^{2}(\Omega) \cap H_{0}^{1}(\Omega)}=-\int_{L^{2}(\partial \Omega)} u \frac{\partial \phi}{\partial \nu \mid \partial \Omega},
$$


for every $u \in L^{2}(\partial \Omega)$, and every $\phi \in H^{2}(\Omega) \cap H_{0}^{1}(\Omega)$.

Then, $B$ is an admissible control operator, if and only if, $B^{*}$ is an admissible observation operator, if and only if, for every $T>0$, there exists $C_{T}>0$ such that, for every $\psi_{0} \in H^{2}(\Omega) \cap H_{0}^{1}(\Omega)$, the solution of

$$
\begin{aligned}
\dot{\psi} & =\triangle \psi \quad \text { in } \Omega, \\
\psi_{\partial \Omega} & =0, \\
\psi(0) & =\psi_{0},
\end{aligned}
$$

satisfies

$$
\int_{0}^{T}\left\|\frac{\partial \psi}{\partial \nu \mid \partial \Omega}(t)\right\|_{L^{2}(\partial \Omega)}^{2} d t \leq C_{T}\left\|\psi_{0}\right\|_{L^{2}(\Omega)}^{2} .
$$

This inequality indeed holds: this is a classical trace regularity result.

Another typical example is provided by second-order equations. The framework is the following (see $[31,32,33])$. Let $H$ be a Hilbert space, and $A_{0}$ : $D\left(A_{0}\right) \rightarrow H$ be selfadjoint and strictly positive. Recall that $D\left(A_{0}^{1 / 2}\right)$ is the completion of $D\left(A_{0}\right)$ with respect to the norm

$$
\|y\|_{D\left(A_{0}^{1 / 2}\right)}=\sqrt{\left\langle A_{0} y, y\right\rangle_{H}},
$$

and that

$$
D\left(A_{0}\right) \subset D\left(A_{0}^{1 / 2}\right) \subset H,
$$

with continuous and dense embeddings. Set

$$
X=D\left(A_{0}^{1 / 2}\right) \times H,
$$

and define $A: D(A) \rightarrow X$ on

$$
D(A)=D\left(A_{0}\right) \times D\left(A_{0}^{1 / 2}\right),
$$

by

$$
A=\left(\begin{array}{cc}
0 & I \\
-A_{0} & 0
\end{array}\right) .
$$

Note that $A$ is skew-adjoint in $X$.

Let $B_{0} \in L\left(U, D\left(A_{0}^{1 / 2}\right)^{\prime}\right.$, where $U$ is a Hilbert, and $D\left(A_{0}^{1 / 2}\right)^{\prime}$ is the dual of $D\left(A_{0}^{1 / 2}\right)$ with respect to the pivot space $U$. We investigate the second-order control system

$$
\begin{aligned}
& y_{t t}+A_{0} y=B_{0} u, \\
& y(0)=y_{0}, y_{t}(0)=y_{1} .
\end{aligned}
$$

It can be written in the form

$$
\frac{\partial}{\partial t}\left(\begin{array}{c}
y \\
y_{t}
\end{array}\right)=A\left(\begin{array}{c}
y \\
y_{t}
\end{array}\right)+B u
$$


where

$$
B=\left(\begin{array}{c}
0 \\
B_{0}
\end{array}\right)
$$

One has

$$
X_{-1}=D\left(A^{*}\right)^{\prime}=H \times D\left(A_{0}^{1 / 2}\right)^{\prime},
$$

with respect to the pivot space $X$, where $D\left(A_{0}^{1 / 2}\right)^{\prime}$ is the dual of $D\left(A_{0}^{1 / 2}\right)$ with respect to the pivot space $H$. Moreover, $B \in L\left(U, H \times D\left(A_{0}^{1 / 2}\right)^{\prime}\right)$, and $B^{*} \in$ $L\left(D\left(A_{0}\right) \times D\left(A_{0}^{1 / 2}\right), U\right)$ is given by

$$
B^{*}=\left(\begin{array}{c}
0 \\
B_{0}^{*}
\end{array}\right) .
$$

Proposition 2.2. The following statements are equivalent:

- $B$ is admissible;

- There exists $C_{T}>0$ such that every solution of

$$
\begin{aligned}
& \psi_{t t}+A_{0} \psi=0, \\
& \psi(0)=\psi_{0} \in D\left(A_{0}\right), \psi_{t}(0)=\psi_{1} \in D\left(A_{0}^{1 / 2}\right),
\end{aligned}
$$

satisfies

$$
\int_{0}^{T}\left\|B_{0}^{*} \psi_{t}(t)\right\|_{U}^{2} d t \leq C_{T}\left(\left\|\psi_{0}\right\|_{D\left(A_{0}^{1 / 2}\right)}^{2}+\left\|\psi_{1}\right\|_{H}^{2}\right)
$$

- There exists $C_{T}>0$ such that every solution of

$$
\begin{aligned}
& \psi_{t t}+A_{0} \psi=0, \\
& \psi(0)=\psi_{0} \in H, \psi_{t}(0)=\psi_{1} \in D\left(A_{0}^{1 / 2}\right)^{\prime},
\end{aligned}
$$

satisfies

$$
\int_{0}^{T}\left\|B_{0}^{*} \psi(t)\right\|_{U}^{2} d t \leq C_{T}\left(\left\|\psi_{0}\right\|_{H}^{2}+\left\|\psi_{1}\right\|_{D\left(A_{0}^{1 / 2}\right)^{\prime}}^{2}\right) .
$$

Example 2.2. Consider the boundary controlled wave equation

$$
\begin{aligned}
y_{t t} & =\triangle y \quad \text { in } \Omega, \\
y_{\mid \partial \Omega} & =u(t) .
\end{aligned}
$$

Set $H=H^{-1}(\Omega)$, and consider the operator

$$
A_{0}=-\triangle: D\left(A_{0}\right)=H_{0}^{1}(\Omega) \rightarrow H .
$$

Then, $A_{0}$ is an isomorphism from $D\left(A_{0}\right)$ in $H$, and

$$
D\left(A_{0}^{1 / 2}\right)=L^{2}(\Omega)
$$


and the dual space $\left(D\left(A_{0}^{1 / 2}\right)\right)^{\prime}$ (with respect to the pivot space $H=H^{-1}(\Omega)$ ) is equal to the dual space $\left(H^{2}(\Omega) \cap H_{0}^{1}(\Omega)\right)^{\prime}$ (with respect to the pivot space $L^{2}(\Omega)$. Indeed, the operator $A_{0}$ can be extended as an operator $A_{-1}: L^{2}(\Omega) \rightarrow$ $\left(H^{2}(\Omega) \cap H_{0}^{1}(\Omega)\right)^{\prime}$. On the other part, set

$$
U=L^{2}(\partial \Omega)
$$

and

$$
X=D\left(A_{0}^{1 / 2}\right) \times H=L^{2}(\Omega) \times H^{-1}(\Omega) .
$$

Then, the controlled wave equation writes

$$
y_{t t}=-A_{0} y+B_{0} u \quad \text { in }\left(D\left(A_{0}^{1 / 2}\right)\right)^{\prime},
$$

where

$$
B_{0}^{*} \phi=\frac{\partial}{\partial \nu}\left(A_{0}^{-1} \phi\right)_{\mid \partial \Omega}
$$

for every $\phi \in L^{2}(\Omega)$.

It is known (see $[17,18,19])$ that there exists $C_{T}>0$ such that

$$
\int_{0}^{T}\left\|\frac{\partial \psi}{\partial \nu}(t)\right\|_{L^{2}(\partial \Omega)}^{2} d t \leq C_{T}\left(\left\|\psi_{0}\right\|_{H_{0}^{1}(\Omega)}^{2}+\left\|\psi_{1}\right\|_{L^{2}(\Omega)}^{2}\right)
$$

for every $\psi \in C^{0}\left(0, T ; H^{2}(\Omega)\right) \cap C^{1}\left(0, T ; H^{1}(\Omega)\right)$ solution of

$$
\begin{aligned}
& \psi_{t t}=\Delta \psi \quad \text { in } \Omega \\
& \psi_{\partial \Omega}=0, \\
& \psi(0)=\psi_{0}, \dot{p s i}(0)=\psi_{1} .
\end{aligned}
$$

Therefore, the observation operator $B^{*}$ (and thus, the control operator $B$ ) is admissible.

Note that $B_{0} \in L\left(U, D\left(A_{0}^{1 / 2}\right)^{\prime}\right)$ is given by

$$
B_{0} u=A_{-1} D u
$$

for every $u \in U$, where $D$ is the Dirichlet mapping, defined by transposition by

$$
\int_{\Omega} D u(x) f(x) d x=\int_{\partial \Omega} u(x) \frac{\partial \phi}{\partial \nu}(x) d x
$$

for all $f$ and $\phi$ so that

$$
\begin{aligned}
& \triangle \phi=f \quad \text { in } \Omega, \\
& \phi_{\partial \Omega}=0 .
\end{aligned}
$$




\subsubsection{Necessary and sufficient conditions for controllability in Ba- nach spaces}

We first recall the notations.

Let $X$ be a Banach space. For clarity, denote by \|\|$_{X}$ the norm of $X$. Let $S(t)$ denote a strongly continuous semigroup on $X$, of generator $(A, D(A))$. Let $X_{-1}$ denote the completion of $X$ for the norm $\|x\|_{-1}=\left\|(\beta I-A)^{-1} x\right\|$, where $\beta \in \rho(A)$ is fixed. The space $X_{-1}$ is isomorphic to $\left(D\left(A^{*}\right)\right)^{\prime}$. The semigroup $S(t)$ extends to a semigroup on $X_{-1}$, still denoted $S(t)$, whose generator is an extension of the operator $A$, still denoted $A$. With these notations, $A$ is a linear operator from $X$ to $X_{-1}$.

Let $U$ be a Banach space. Denote by \|\|$_{U}$ the associated norm. Let $B \in$ $L\left(U, X_{-1}\right)$ be and admissible control operator. Consider the control system

$$
\dot{y}(t)=A y(t)+B u(t)
$$

with $y(0)=y_{0} \in X$ and $u(\cdot) \in L^{2}(0,+\infty ; U)$. The solution writes

$$
y(t)=S(t) y_{0}+\int_{0}^{t} S(t-s) B u(s) d s
$$

for every $t \geq 0$. For $T>0$, the operator $L_{T}: L^{2}(0, T ; U) \rightarrow X_{-1}$ is defined by

$$
L_{T} u=\int_{0}^{T} S(t-s) B u(s) d s .
$$

Note that, since $B$ is admissible, $L_{T} \in L\left(L^{2}(0, T ; U), X\right)$.

Definition 2.3. For $y_{0} \in X$, and $T>0$, the system (10) is said to be exactly controllable from $y_{0}$ in time $T$ if, for every $y_{1} \in X$, there exists $u(\cdot) \in L^{2}(0, T ; U)$ so that the solution of $(10)$, with $y(0)=y_{0}$, associated with the control $u(\cdot)$, satisfies $y(T)=y_{1}$.

It is clear that the system (10) is exactly controllable from $y_{0}$ in time $T$ if and only if $L_{T}$ is onto, that is $\operatorname{Im} L_{T}=X$. In particular, if the system (10) is exactly controllable from $y_{0}$ in time $T$, then it is exactly controllable from any point $y_{0}^{\prime} \in X$ in time $T$. One says that the system (10) is exactly controllable in time $T$.

Definition 2.4. The system (10) is said to be approximately controllable from $y_{0}$ in time $T$ if, for every $y_{1} \in X$ and every $\varepsilon>0$, there exists $u(\cdot) \in L^{2}(0, T ; V)$ so that the solution of $(10)$, with $y(0)=y_{0}$, associated with the control $u(\cdot)$, satisfies $\left\|y(T)-y_{1}\right\|_{X} \leq \varepsilon$.

As previously, this notion does not depend on the initial point, and the system (10) is approximately controllable in time $T$ if and only if $\operatorname{Im} L_{T}$ is dense in $X$.

Definition 2.5. For $T>0$, the system (10) is said to be exactly null controllable in time $T$ if, for every $y_{0} \in X$, there exists $u(\cdot) \in L^{2}(0, T ; U)$ so that the solution of (10), with $y(0)=y_{0}$, associated with the control $u(\cdot)$, satisfies $y(T)=0$. 
Remark 2.1. If the system (17) is exactly null controllable in every time $T$, then it is approximately controllable in every time $T$.

Theorem 2.3. - The system (17) is exactly controllable in time $T$ if and only if there exists $\alpha>0$ so that

$$
\int_{0}^{T}\left\|B^{*} S^{*}(t) \psi\right\|_{U}^{2} d t \geq \alpha\|\psi\|_{X}^{2}
$$

for every $\psi \in D\left(A^{*}\right)$ (observability inequality). This is equivalent to saying that $L_{T}^{*}$ is bounded below.

- The system (17) is approximately controllable in time $T$ if and only if the following implication holds:

$$
\forall t \in[0, T] \quad B^{*} S^{*}(t) \psi=0 \Rightarrow \psi=0,
$$

for every $\psi \in D\left(A^{*}\right)$. This is equivalent to saying that $L_{T}^{*}$ is one-to-one.

- The system (17) is exactly null controllable in time $T$ if and only if there exists $\alpha>0$ so that

$$
\int_{0}^{T}\left\|B^{*} S^{*}(t) \psi\right\|_{U}^{2} d t \geq \alpha\left\|S(T)^{*} \psi\right\|_{X}^{2}
$$

for every $\psi \in D\left(A^{*}\right)$. This is equivalent to saying that $\operatorname{Im} S(T) \subset \operatorname{Im} L_{T}$.

Remark 2.2. Assume that $B$ is admissible and that the control system (10) is exactly null controllable in time $T$. Let $y_{0} \in X$. For every $\psi \in D\left(A^{*}\right)$, set

$$
J(\psi)=\frac{1}{2} \int_{0}^{T}\left\|B^{*} S(t)^{*} \psi\right\|_{U}^{2} d t+\left\langle S\left(T^{*}\right) \psi, y_{0}\right\rangle_{X} .
$$

The functional $J$ is strictly convex, and, from the observability inequality (14), is coercive. Define the control $u$ by

$$
u(t)=B^{*} S(T-t)^{*} \psi
$$

for every $t \in[0, T]$, and let $y(\cdot)$ be the solution of $(10)$, such that $y(0)=y_{0}$, associated with the control $u$. Then, one has $y(T)=0$, and moreover, $u$ is the control of minimal $L^{2}$ norm, among all controls whose associated trajectory satisfies $y(T)=0$.

This remark proves that observability implies controllability, and gives a constructive way to build the control of minimal $L^{2}$ norm (see [38]). This is more or less the contents of the Hilbert Uniqueness Method (see [17, 18]). Hence, in what follows, we refer to the control (16) as the HUM control.

The same remark holds of course for exact controllability, with the functional

$$
J(\psi)=\frac{1}{2} \int_{0}^{T}\left\|B^{*} S(t)^{*} \psi\right\|_{U}^{2} d t-\left\langle\psi, y_{1}\right\rangle_{X}+\left\langle S(T)^{*} \psi, y_{0}\right\rangle_{X} .
$$


Example 2.3. For the heat equation of Example 2.1,

$$
\begin{aligned}
\dot{y} & =\triangle y \quad \text { in } \Omega, \\
y_{\mid \partial \Omega} & =u(t), \\
y(0) & =y_{0} \in L^{2}(\Omega),
\end{aligned}
$$

it follows from $[8,15]$ that, for every $T>0$, there exists $c_{T}>0$ so that, for every $\psi_{0} \in H^{2}(\Omega) \cap H_{0}^{1}(\Omega)$, the solution of

$$
\begin{aligned}
\dot{\psi} & =\triangle \psi \quad \text { in } \Omega, \\
\psi_{\mid \partial \Omega} & =0, \\
\psi(0) & =\psi_{0},
\end{aligned}
$$

satisfies

$$
\int_{0}^{T}\left\|\frac{\partial \psi}{\partial \nu} \mid \partial \Omega(t)\right\|_{L^{2}(\partial \Omega)}^{2} d t \geq c_{T}\left\|\psi_{0}\right\|_{L^{2}(\Omega)}^{2} .
$$

In other words, the heat equation with boundary control is exactly null controllable, in any time $T>0$.

Example 2.4. Consider the heat equation with distributed control

$$
\begin{aligned}
\dot{y} & =\triangle y+1_{\mathcal{O}} u \quad \text { in } \Omega, \\
y_{\mid \partial \Omega} & =0, \\
y(0) & =y_{0} \in L^{2}(\Omega),
\end{aligned}
$$

where $\mathcal{O}$ is an open subset of $\Omega$. It follows from $[8,15]$ that, for every $T>0$, there exists $c_{T}>0$ so that, for every $\psi_{0} \in L^{2}(\Omega)$, the solution of

$$
\begin{aligned}
\dot{\psi} & =\triangle \psi \quad \text { in } \Omega, \\
\psi_{\mid \partial \Omega} & =0, \\
\psi(0) & =\psi_{0},
\end{aligned}
$$

satisfies

$$
\int_{0}^{T} \int_{\mathcal{O}} \psi(t, x)^{2} d x d t \geq c_{T} \int_{\Omega} \psi(T, x)^{2} d x .
$$

In other words, the heat equation with distributed control is exactly null controllable (in the space $L^{2}(\Omega)$, in any time $T>0$.

Note that these observability inequalities are proved in $[8,15]$ using Carleman estimates. In both cases, note also that Holmgren's Uniqueness Theorem implies approximate controllability in $L^{2}(\Omega)$.

Example 2.5. Consider the wave equation of Example 2.2. It is proved in [3] that it is exactly controllable, under the so-called GCC (Geometric Control Condition), within time $T$ sufficiently large. The time of controllability has to be large enough, because of the finite speed of propagation of the wave equation. The observability inequality has been proved in 
- [7], for $T$ large enough, with a condition on $\partial \Omega$, using multipliers methods;

- $[11,18]$, for $T$ large enough, using multipliers methods;

- [3], using microlocal analysis;

and in many other references.

Note that, in dimension one, the proof of the observability inequality can be achieved easily using Fourier series, for $T \geq 2 L$, where $L$ is the length of the interval.

The observability inequality implies a result of exact controllability in the space $L^{2}(\Omega) \times H^{-1}(\Omega)$.

\section{Semidiscrete approximations of infinite dimen- sional linear control systems in Hilbert spaces}

\subsection{Introduction}

Consider the infinite dimensional linear control system

$$
\begin{aligned}
\dot{y}(t) & =A y(t)+B u(t), \\
y(0) & =y_{0},
\end{aligned}
$$

where the state $y(t)$ belongs to a Hilbert space $X$, the control $u(t)$ belongs to a Hilbert space $U, A: D(A) \rightarrow X$ is an operator, and $B$ is a control operator (in general, unbounded) on $U$. Discretizing this partial differential equation, using for instance a finite difference, or a finite element scheme, leads to a family of finite dimensional linear control systems

$$
\begin{aligned}
\dot{y}_{h}(t) & =A y_{h}(t)+B u_{h}(t), \\
y(0) & =y_{0 h},
\end{aligned}
$$

where $y_{h}(t) \in X_{h}$ and $u_{h}(t) \in U_{h}$, for $0<h<h_{0}$.

Let $y_{1} \in X$; if the control system (17) is controllable in time $T$, then there exists a solution $y(\cdot)$ of $(17)$, associated with a control $u$, such that $y(T)=y_{1}$. We address the following question: is it possible to find controls $u_{h}$, for $0<h<$ $h_{0}$, converging to the control $u$ as the mesh size $h$ of the discretization process tends to zero, and such that the associated trajectories $y_{h}(\cdot)$, solutions of (18), converge to $y(\cdot)$ ? Moreover, does there exist an efficient algorithmic way to determine the controls $u_{h}$ ?

For controllable linear control systems of the type (17), we have available many methods in order to realize the controllability. Among them, the Hilbert Uniqueness Method (HUM), introduced in [17, 18], is adapted to numerical implementations. It consists in minimizing a cost function, namely, the $L^{2}$ norm of the control. In Section 3.2, we answer to the above question in the case where controllability of (17) is achieved using the HUM method. The objective is to establish conditions ensuring uniform controllability of the family of discretized 
control systems (18), and to establish a computationally feasible approximation method for realizing controllability.

The question of uniform controllability and/or observability of the family of approximation control systems (18) has been investigated by E. Zuazua and collaborators in a series of articles [5, 9, 16, 21, 22, 24, 30, 36, 37, 38, 39], for different discretization processes, on different examples. When the observability constant of the finite dimensional approximation systems does not depend on $h$, one says that the property of uniform observability holds. For classical finite difference schemes, a uniform boundary observability property holds for one dimensional heat equations [21], beam equations [16], Schrödinger equations [39], but does not hold for 1-D wave equations [9]. In this latter case, the observability constant of the one-dimensional semidiscretized wave equation tends to infinity as the mesh size tends to zero. This is due to a pathological behavior of high frequency eigenvalues of the semidiscrete model. Actually, spurious oscillations appear, due to interferences with the mesh, that are responsible for non uniformity. From the point of view of controllability, they cause divergence of controls as the mesh size is tending to zero. These results hold for other numerical schemes, such as the classical $P_{1} \times P_{1}$ finite elements method, and also for two-dimensional linear wave equations (see $[9,22,36]$ ). In the case of wave equations, several remedies are provided to reestablish uniformity: cutting off high frequencies by Fourier filtering; Tychonoff regularization, which consists in adding a viscosity term to the semidiscrete model; two-grid algorithms, which consist in using different sized grids for filtering solutions; the use of mixed finite elements, namely, $P_{1} \times P_{0}$ finite elements. This latter method, used in [5], is interesting from the practical point of view, because it is simple to implement, and does not require any further filtering procedure or extra corrections. Moreover, from the theoretical point of view it seems natural because it takes into account the natural difference of regularity between $u$ and $u_{t}$, for the wave equation. The case of the wave equation is hence quite involved. In contrast, it seems that, for 1-D heat, beam and Schrödinger equations, the dissipative and/or dispersive effects help to recover some uniformity.

The HUM method is not the unique method to discretize the control. We can imagine other ways to realize the controllability for (18), with the property $u_{h} \rightarrow u$. Related to this problem is the problem of uniform stabilizability. The question is the following: is it true that (17) is stabilizable if and only if (18) is uniformly stabilizable?

Recall that (17) is stabilizable if there exists $K \in L(X, U)$ so that $A+B K$ generates an exponentially stable semigroup $S(t)$, that is,

$$
\exists M, \omega>0 \mid \forall t \geq 0 \quad\|S(t)\| \leq M \mathrm{e}^{-\omega t} .
$$

On the other part, (18) is said uniformly stabilizable if, for every $h \in\left(0, h_{0}\right)$, there exists a $(m \times n)$ matrix $K_{h}$ such that the matrix $A_{h}+B_{h} K_{h}$ is uniformly exponentially stable, that is,

$$
\exists M, \omega>0 \mid \forall t \geq 0 \quad \forall h \in\left(0, h_{0}\right) \quad\left\|\mathrm{e}^{t\left(A_{h}+B_{h} K_{h}\right)}\right\| \leq M \mathrm{e}^{-\omega t} .
$$


This question has been widely investigated, in particular, in the context of the Riccati theory. In $[1,2,6,10,14,20,26]$, approximation results are provided for the linear quadratic regulator $(L Q R)$ problem in the parabolic case, or in the hyperbolic damped case, that show, in particular, the convergence of the controls of the semidiscrete models to the control of the continuous model. However, in the LQR problem, the final point is not fixed. The exact controllability problem is a very different matter. Actually, it appears from the discussion above that the divergence of the controls as the mesh size tends to zero in the exact controllability problem is due to is the requirement to drive the final state exactly to a given point.

Note that, as expected, this problem disappears for the approximate controllability problem, which can be seen as a relaxation of the exact controllability problem (see [38]).

\subsection{Uniform controllability of semidiscrete approximations of parabolic control systems}

We saw previously that controlling an approximation model of a controllable infinite dimensional linear control system does not necessarily yield a good approximation of the control needed for the continuous model. In this section, we report on recent results obtained in [12], in which it is proved that, under the main assumptions that the discretized semigroup is uniformly analytic, and that the control operator is mildly unbounded, the semidiscrete approximation models are uniformly controllable.

The discretization framework used here is in the same spirit as the one of $[1,2,6,10,14,20,26]$.

The question of uniform controllability of the discretized models (18) is investigated in the case where the operator $A$ generates an analytic semigroup. Of course, due to regularization properties, the control system (17) is not exactly controllable in general. Hence, we focus on exact null controllability. The main result, Theorem 3.1, states that, for an exactly null controllable parabolic system (17), under standard assumptions on the discretization process (that are satisfied for most of classical schemes), if the discretized semigroup is uniformly analytic (see [14]), and if the degree of unboundedness of the control operator $B$ with respect to $A$ (see [27]) is lower than $1 / 2$, then the approximating control systems are uniformly controllable. A uniform observability and admissibility inequality is proved. Moreover, we provide a minimization procedure to compute the approximation controls. Note that this condition on the degree of unboundedness of $B$ is satisfied for distributed controls (that is, if $B$ is bounded), and, if $B$ is unbounded, it is for instance satisfied for the heat equation with Neumann boundary control, but not with Dirichlet boundary control.

The precise results are as follows.

Let $X$ and $U$ be Hilbert spaces, and let $A: D(A) \rightarrow X$ be a linear operator, generating a strongly continuous semigroup $S(t)$ on $X$. Let $B \in L\left(U, D\left(A^{*}\right)^{\prime}\right)$ be a control operator. We make the following assumptions. 
$\left(\mathbf{H}_{\mathbf{1}}\right)$ The semigroup $S(t)$ is analytic.

Therefore (see [25]), there exist positive real numbers $C_{1}$ and $\omega$ such that

$$
\|S(t) y\|_{X} \leq C_{1} \mathrm{e}^{\omega t}\|y\|_{X} \text {, and }\|A S(t) y\|_{X} \leq C_{1} \frac{\mathrm{e}^{\omega t}}{t}\|y\|_{X},
$$

for all $t>0$ and $y \in D(A)$, and such that, if we set

$$
\hat{A}=A-\omega I,
$$

then the fractional powers $(-\hat{A})^{\theta}$ of $\hat{A}$ are well defined, for $\theta \in[0,1]$, and there holds

$$
\left\|(-\hat{A})^{\theta} S(t) y\right\|_{X} \leq C_{1} \frac{\mathrm{e}^{\omega t}}{t^{\theta}}\|y\|_{X}
$$

for all $t>0$ and $y \in D(A)$.

Of course, inequalities (19) hold as well if one replaces $A$ by $A^{*}, S(t)$ by $S(t)^{*}$, for $y \in D\left(A^{*}\right)$.

Moreover, if $\rho(A)$ denotes the resolvent set of $A$, then there exists $\delta \in(0, \pi / 2)$ such that

$$
\rho(A) \supset \Delta_{\delta}=\left\{\omega+\rho \mathrm{e}^{i \theta}|\rho>0,| \theta \mid \leq \frac{\pi}{2}+\delta\right\} .
$$

For $\lambda \in \rho(A)$, denote by $R(\lambda, A)=(\lambda I-A)^{-1}$ the resolvent of $A$. It follows from the previous estimates that there exists $C_{2}>0$ such that

$$
\|R(\lambda, A)\|_{L(X)} \leq \frac{C_{2}}{|\lambda-\omega|}, \text { and }\|A R(\lambda, A)\|_{L(X)} \leq C_{2},
$$

for every $\lambda \in \Delta_{\delta}$, and

$$
\|R(\lambda, \hat{A})\|_{L(X)} \leq \frac{C_{2}}{|\lambda|} \text {, and }\|\hat{A} R(\lambda, \hat{A})\|_{L(X)} \leq C_{2},
$$

for every $\lambda \in\left\{\Delta_{\delta}+\omega\right\}$. Similarly, inequalities (23) and (23) hold as well with $A^{*}$ and $\hat{A}^{*}$.

$\left(\mathbf{H}_{2}\right)$ The degree of unboundedness of $B$ is lower than $1 / 2$. In other words, there exists $\gamma \in[0,1 / 2)$ such that

$$
B \in L\left(U, D\left(\left(-\hat{A}^{*}\right)^{\gamma}\right)^{\prime}\right) .
$$

In these conditions, the domain of $B^{*}$ is $D\left(B^{*}\right)=D\left(\left(-\hat{A}^{*}\right)^{\gamma}\right)$. Moreover, there exists a constant $C_{3}>0$ such that

$$
\left\|B^{*} \psi\right\|_{U} \leq C_{3}\left\|\left(-\hat{A}^{*}\right)^{\gamma} \psi\right\|_{X},
$$

for every $\psi \in D\left(\left(-\hat{A}^{*}\right)^{\gamma}\right)$.

Note that this assumption implies that the control operator $B$ is admissible.

We next introduce adapted approximation assumptions, inspired by [14] (see also $[1,2,6,10,20,26])$. Consider two families $\left(X_{h}\right)_{0<h<h_{0}}$ and $\left(U_{h}\right)_{0<h<h_{0}}$ of finite dimensional spaces, where $h$ is the discretization parameter. 
$\left(\mathbf{H}_{3}\right)$ For every $h \in\left(0, h_{0}\right)$, there exist linear mappings $P_{h}: D\left(\left(-\hat{A}^{*}\right)^{1 / 2}\right)^{\prime} \rightarrow X_{h}$ and $\widetilde{P}_{h}: X_{h} \rightarrow D\left(\left(-\hat{A}^{*}\right)^{1 / 2}\right)$ (resp., there exist linear mappings $Q_{h}: U \rightarrow$ $U_{h}$ and $\left.\widetilde{Q}_{h}: U_{h} \rightarrow U\right)$, satisfying the following requirements:

$\left(\mathbf{H}_{\mathbf{3 . 1}}\right)$ For every $h \in\left(0, h_{0}\right)$, there holds

$$
P_{h} \widetilde{P}_{h}=i d_{X_{h}} \text {, and } Q_{h} \widetilde{Q}_{h}=i d_{U_{h}} .
$$

$\left(\mathbf{H}_{3.2}\right)$ There exist $s>0$ and $C_{4}>0$ such that there holds, for every $h \in$ $\left(0, h_{0}\right)$,

$$
\begin{gathered}
\left\|\left(I-\widetilde{P}_{h} P_{h}\right) \psi\right\|_{X} \leq C_{4} h^{s}\left\|A^{*} \psi\right\|_{X}, \\
\left\|\left(-\hat{A}^{*}\right)^{\gamma}\left(I-\widetilde{P}_{h} P_{h}\right) \psi\right\|_{X} \leq C_{4} h^{s(1-\gamma)}\left\|A^{*} \psi\right\|_{X},
\end{gathered}
$$

for every $\psi \in D\left(A^{*}\right)$, and

$$
\left\|\left(I-\widetilde{Q}_{h} Q_{h}\right) u\right\|_{U} \underset{h \rightarrow 0}{\longrightarrow} 0,
$$

for every $u \in U$, and

$$
\left\|\left(I-\widetilde{Q}_{h} Q_{h}\right) B^{*} \psi\right\|_{U} \leq C_{4} h^{s(1-\gamma)}\left\|A^{*} \psi\right\|_{X}
$$

for every $\psi \in D\left(A^{*}\right)$.

Note that (29) makes sense since, by assumption, $\gamma<1 / 2$, and thus, $\operatorname{Im} \widetilde{P}_{h} \subset D\left(\left(-\hat{A}^{*}\right)^{1 / 2}\right) \subset D\left(\left(-\hat{A}^{*}\right)^{\gamma}\right)$.

For every $h \in\left(0, h_{0}\right)$, the vector space $X_{h}$ (resp. $\left.U_{h}\right)$ is endowed with the norm \|\|$_{X_{h}}$ (resp., \|\|$_{U_{h}}$ ) defined by

$$
\left\|y_{h}\right\|_{X_{h}}=\left\|\widetilde{P}_{h} y_{h}\right\|_{X},
$$

for $y_{h} \in X_{h}$ (resp., $\left\|u_{h}\right\|_{U_{h}}=\left\|\widetilde{Q}_{h} u_{h}\right\|_{U}$, for $u_{h} \in U_{h}$ ). In these conditions, it is clear that

$$
\left\|\widetilde{P}_{h}\right\|_{L\left(X_{h}, X\right)}=\left\|\widetilde{Q}_{h}\right\|_{L\left(U_{h}, U\right)}=1,
$$

for every $h \in\left(0, h_{0}\right)$. Moreover, it follows from (28), (29), (30), and from the Banach-Steinhaus Theorem, that there exists $C_{5}>0$ such that

$$
\left\|P_{h}\right\|_{L\left(X, X_{h}\right)} \leq C_{5}, \text { and }\left\|Q_{h}\right\|_{L\left(U, U_{h}\right)} \leq C_{5},
$$

and

$$
\left\|\left(-\hat{A}^{*}\right)^{\gamma}\left(I-\widetilde{P}_{h} P_{h}\right) \psi\right\|_{X} \leq C_{5}\left\|\left(-\hat{A}^{*}\right)^{\gamma} \psi\right\|_{X},
$$

for every $h \in\left(0, h_{0}\right)$, and every $\psi \in D\left(\left(-\hat{A}^{*}\right)^{\gamma}\right)$. 
$\left(\mathbf{H}_{3.3}\right)$ For every $h \in\left(0, h_{0}\right)$, there holds

$$
P_{h}=\widetilde{P}_{h}^{*}, \text { and } Q_{h}=\widetilde{Q}_{h}^{*},
$$

where the adjoint operators are considered with respect to the pivot spaces $X, U, X_{h}$, and $U_{h}$.

Note that this assumption indeed holds for most of classical schemes

(Galerkin or spectral approximations, centered finite differences, ...).

$\left(\mathbf{H}_{3.4}\right)$ There exists $C_{6}>0$ such that

$$
\left\|B^{*} \widetilde{P}_{h} \psi_{h}\right\|_{U} \leq C_{6} h^{-\gamma s}\left\|\psi_{h}\right\|_{X_{h}},
$$

for all $h \in\left(0, h_{0}\right)$ and $\psi_{h} \in X_{h}$.

For every $h \in\left(0, h_{0}\right)$, we define the approximation operators $A_{h}^{*}: X_{h} \rightarrow X_{h}$ of $A^{*}$, and $B_{h}^{*}: X_{h} \rightarrow U_{h}$ of $B^{*}$, by

$$
A_{h}^{*}=P_{h} A^{*} \widetilde{P}_{h}, \text { and } B_{h}^{*}=Q_{h} B^{*} \widetilde{P}_{h} .
$$

Due to Assumption $\left(H_{3.3}\right)$, it is clear that $B_{h}=P_{h} B \widetilde{Q}_{h}$, for every $h \in\left(0, h_{0}\right)$. On the other part, we set $A_{h}=\left(A_{h}^{*}\right)^{*}$ (with respect to the pivot space $X_{h}$ ). Note that, if $A$ is selfadjoint, then $A_{h}=P_{h} A \widetilde{P}_{h}$.

$\left(\mathbf{H}_{4}\right)$ The following properties hold:

$\left(\mathbf{H}_{4.1}\right)$ The family of operators $\mathrm{e}^{t A_{h}^{*}}$ is uniformly analytic, in the sense that there exists $C_{7}>0$ such that

$$
\left\|\mathrm{e}^{t A_{h}}\right\|_{L\left(X_{h}\right)} \leq C_{7} \mathrm{e}^{\omega t}, \text { and }\left\|A_{h} \mathrm{e}^{t A_{h}}\right\|_{L\left(X_{h}\right)} \leq C_{7} \frac{\mathrm{e}^{\omega t}}{t}
$$

for every $t>0$.

Under this assumption, there exists $C_{8}>0$ such that

$$
\left\|R\left(\lambda, A_{h}\right)\right\|_{L\left(X_{h}\right)} \leq \frac{C_{8}}{|\lambda-\omega|},
$$

for every $\lambda \in \Delta_{\delta}$. Note that (39) and (40) hold as well if one replaces $A_{h}$ with $A_{h}^{*}$.

$\left(\mathbf{H}_{4.2}\right)$ There exists $C_{9}>0$ such that, for every $f \in X$ and every $h \in\left(0, h_{0}\right)$, the respective solutions of $\hat{A}^{*} \psi=f$ and $\hat{A}_{h}^{*} \psi_{h}=P_{h} f$ satisfy

$$
\left\|P_{h} \psi-\psi_{h}\right\|_{X_{h}} \leq C_{9} h^{s}\|f\|_{X}
$$


In other words, there holds $\left\|P_{h} \hat{A}^{*-1}-\hat{A}_{h}^{*-1} P_{h}\right\|_{L\left(X, X_{h}\right)} \leq C_{9} h^{s}$. This is a (strong) rate of convergence assumption.

Remark 3.1. Assumptions $\left(H_{3}\right)$ and $\left(H_{4.2}\right)$ hold for most of the classical numerical approximation schemes, such as Galerkin methods, spectral methods, centered finite difference schemes, ... As noted in [14], Assumption $\left(H_{4.1}\right)$ of uniform analyticity is not standard, and has to be checked in each specific case. However, it can be shown to hold, under Assumption $\left(H_{1}\right)$, provided the bilinear form associated with $A_{h}$ is uniformly coercive (see [4] for the selfadjoint case, and [13, Lemma 4.2] for the general nonselfadjoint case).

Theorem 3.1. Under the previous assumptions, the control system $\dot{y}=A y+B u$ is exactly null controllable in time $T>0$, if and only if the family of discretized control systems $\dot{y}_{h}=A_{h} y_{h}+B_{h} u_{h}$ is uniformly controllable in the following sense. There exist $\beta>0, h_{1}>0$, and positive real numbers $c, c^{\prime}$, such that the uniform observability and admissibility inequality

$$
c\left\|\mathrm{e}^{T A_{h}^{*}} \psi_{h}\right\|_{X_{h}}^{2} \leq \int_{0}^{T}\left\|B_{h}^{*} \mathrm{e}^{t A_{h}^{*}} \psi_{h}\right\|_{U_{h}}^{2} d t+h^{\beta}\left\|\psi_{h}\right\|_{X_{h}}^{2} \leq c^{\prime}\left\|\psi_{h}\right\|_{X_{h}}^{2}
$$

holds, for every $h \in\left(0, h_{1}\right)$ and every $\psi_{h} \in X_{h}$.

In these conditions, for every $y_{0} \in X$, and every $h \in\left(0, h_{1}\right)$, there exists a unique $\psi_{h} \in X_{h}$ minimizing the functional

$$
J_{h}\left(\psi_{h}\right)=\frac{1}{2} \int_{0}^{T}\left\|B_{h}^{*} \mathrm{e}^{t A_{h}^{*}} \psi_{h}\right\|_{U_{h}}^{2} d t+\frac{1}{2} h^{\beta}\left\|\psi_{h}\right\|_{X_{h}}^{2}+\left\langle\mathrm{e}^{T A_{h}^{*}} \psi_{h}, P_{h} y_{0}\right\rangle_{X_{h}},
$$

and the sequence of controls $\left(\widetilde{Q}_{h} u_{h}\right)_{0<h<h_{1}}$, where $u_{h}$ is defined by

$$
u_{h}(t)=B_{h}^{*} \mathrm{e}^{(T-t) A_{h}^{*}} \psi_{h},
$$

for every $t \in[0, T]$, converges weakly (up to a subsequence), in the space $L^{2}(0, T ; U)$, to a control $u$ such that the solution of

$$
\dot{y}=A y+B u, y(0)=y_{0},
$$

satisfies $y(T)=0$. For every $h \in\left(0, h_{1}\right)$, let $y_{h}(\cdot)$ denote the solution of

$$
\dot{y}_{h}=A_{h} y_{h}+B_{h} u_{h}, y_{h}(0)=P_{h} y_{0} .
$$

Then,

- $y_{h}(T)=-h^{\beta} \psi_{h}$;

- the sequence $\left(\widetilde{P}_{h} y_{h}(\cdot)\right)_{0<h<h_{1}}$ converges weakly (up to a subsequence), in the space $L^{2}(0, T ; X)$, to $y(\cdot)$ on $[0, T]$; 
- for every $t \in(0, T]$, the sequence $\left(\widetilde{P}_{h} y_{h}(t)\right)_{0<h<h_{1}}$ converges weakly (up to a subsequence), in the space $X$, to $y(t)$.

Furthermore, there holds

$$
\int_{0}^{T}\|u(t)\|_{U}^{2} d t \leq \frac{1}{c}\left\|y_{0}\right\|_{X}^{2},
$$

and there exists $M>0$ such that, for every $h \in\left(0, h_{1}\right)$,

$$
\begin{aligned}
& \int_{0}^{T}\left\|u_{h}(t)\right\|_{U_{h}}^{2} d t \leq M^{2}\left\|y_{0}\right\|_{X}^{2}, \quad h^{\beta}\left\|\psi_{h}\right\|_{X_{h}}^{2} \leq M^{2}\left\|y_{0}\right\|_{X}^{2}, \\
& \quad \text { and }\left\|y_{h}(T)\right\|_{X_{h}} \leq M h^{\beta / 2}\left\|y_{0}\right\|_{X} .
\end{aligned}
$$

Remark 3.2. The left-hand side of (42) is a uniform observability inequality for the control systems $\dot{y}_{h}=A_{h} y_{h}+B_{h} u_{h}$. The right-hand side of that inequality means that the control operators $B_{h}$ are uniformly admissible.

Remark 3.3. A similar result holds if the control system $\dot{y}=A y+B u$ is exactly controllable in time $T>0$. However, due to Assumption $\left(H_{1}\right)$, the semigroup $S(t)$ enjoys in general regularity properties. Therefore, the solution $y(\cdot)$ of the control system may belong to a subspace of $X$, whatever the control $u$ is. For instance, in the case of the heat equation with a Dirichlet or Neumann boundary control, the solution is a smooth function of the state variable $x$, as soon as $t>0$, for every control and initial condition $y_{0} \in L^{2}$. Hence, exact controllability does not hold in this case in the space $L^{2}$ (for results on exact null controllability, see $[8,15])$.

The theorem states that the controls $u_{h}$ defined by (44) tend to a control $u$ realizing the exact null controllability for (45). On may wonder under which assumptions the control $u$ is the HUM control such that $y(T)=0$ (see Remark 2.2). The following result provides an answer.

Proposition 3.2. With the notations of Theorem 3.1, if the sequence of real numbers $\left\|\psi_{h}\right\|_{X_{h}}, 0<h<h_{1}$, is moreover bounded, then the control $u$ is the unique HUM control such that $y(T)=0$.

A sufficient condition on $y_{0} \in X$, ensuring the boundedness of the sequence $\left(\left\|\psi_{h}\right\|_{X_{h}}\right)_{0<h<h_{1}}$, is the following: there exists $\eta>0$ such that the control system $\dot{y}=A y+B u$ is exactly null controllable in time $t$, for every $t \in[T-\eta, T+\eta]$, and the trajectory $t \mapsto S(t) y_{0}$ in $X$, for $t \in[T-\eta, T+\eta]$, is not contained in a hyperplane of $X$.

An example where this situation indeed occurs is the following. Additionally to the previous assumptions, assume that the operator $A$ admits a Hilbertian basis of eigenvectors $e_{k}$, associated with eigenvalues $\lambda_{k}$, for $k \in \mathbb{N}$, satisfying

$$
\sum_{k=1}^{+\infty} \frac{-1}{\lambda_{k}}<+\infty
$$


Let $y_{0}=\sum_{k \in \mathbb{N}} y_{0 k} e_{k}$ a point of $X$ such that $y_{0 k} \neq 0$, for every $k \in \mathbb{N}$. Then, the assumption of Proposition 3.2 is satisfied. Indeed, if the trajectory $t \mapsto S(t) y_{0}$ in $X$, for $t \in[T-\eta, T+\eta]$, were contained in a hyperplane of $X$, there would exist $\Phi=\sum_{k \in \mathbb{N}} \Phi_{k} e_{k} \in X \backslash\{0\}$ so that

$$
\sum_{k \in \mathbb{N}} \mathrm{e}^{\lambda_{k} t} y_{0 k} \Phi_{k}=0,
$$

for every $t \in[T-\eta, T+\eta]$. It is well known that the condition (49) implies that the functions $\mathrm{e}^{\lambda_{k} t}, k \in \mathbb{N}$, are independent in $L^{2}$. Hence, $y_{0 k} \Phi_{k}=0$, for every $k \in \mathbb{N}$. This yields a contradiction.

Conclusion. Under standard assumptions on the discretization process, for an exactly null controllable linear control system, if the semigroup of the approximating system is uniformly analytic, and if the degree of unboundedness of the control operator is lower than $1 / 2$, then the semidiscrete approximation models are uniformly controllable.

The problem of providing rates of convergence for the controls of the semidiscrete models is an open problem.

The condition on the degree of unboundedness $\gamma$ of the control operator $B$ is very stringent, and an interesting open problem is to investigate whether the results of this article still hold whenever $\gamma \geq 1 / 2$. Note that, if $\gamma<1 / 2$, then $B$ is automatically admissible; this does not hold necessarily whenever $\gamma \geq 1 / 2$, and may cause some technical difficulties. However, there are many important and relevant problems for which $\gamma \geq 1 / 2$, that are not covered by the previous result, such as, for instance, the heat equation with Dirichlet boundary control. Note that, in this case, although Theorem 3.1 cannot be applied, the finite difference semidiscrete models are uniformly controllable in the one dimensional case (see $[9])$.

Another open and challenging question, probably much more difficult, is to remove the assumption of uniform analyticity of the discretized semigroup. In the case of the one dimensional wave equation, a result of uniform controllability was proved when using a mixed finite element discretization process (see [5]); the extension to higher dimensions is not clear (see [39]). However, a general result, stating uniform stabilization properties, was derived in [26] for general hyperbolic systems.

Finally, the question of uniform controllability of semidiscrete approximations of controlled partial differential equations is completely open in semilinear (more generally, nonlinear) case. It seems reasonable to investigate, in a first step, whether similar results hold in the case of globally Lipschitzian nonlinearities. Indeed, using fixed point arguments combined with the HUM method (see for instance [37]), it should be possible to reduce the study of the controllability to the linear case. 


\section{References}

[1] H. T. BAnKs AND K. ITO, Approximation in LQR problems for infinite dimensional systems with unbounded input operators, J. Math. Systems Estim. Control, 7 (1997), no. 1.

[2] H. T. BANKS AND K. Kunisch, The linear regulator problem for parabolic systems, SIAM J. Control Optim., 22 (1984), no. 5, pp. 684-698.

[3] C. Bardos, G. LeBeAu And J. RAUCH, Sharp sufficient conditions for the observation, control and stabilization of waves from the boundary, SIAM J. Cont. Optim., 30 (1992), 1024-1065.

[4] J. Bramble, A. Shatz, V. Thomee and L. Wahlbin, Some convergence estimates for semidiscrete Galerkin type approximations for parabolic equations, SIAM J. Num. Anal., 14 (1977), pp. 218-241.

[5] C. Castro And S. Micu, Boundary controllability of a linear semidiscrete 1-D wave equation derived from a mixed finite element method, Preprint Univ. Madrid, 2005.

[6] J. S. Gibson, The Riccati integral equations for optimal control problems on Hilbert spaces, SIAM J. Control Optim., 17 (1979), pp. 537-565.

[7] L. F. Ho, Observabilité frontière de l'équation des ondes, C. R. Acad. Sci. Paris, 302 (1986), 443-446.

[8] O. Yu. Imanuvilov, Controllability of parabolic equations, Sb. Math., 186, 6 (1995), pp. 879-900.

[9] J. A. Infante And E. ZuAZUA, Boundary observability for the space semi-discretizations of the 1-D wave equation, M2AN Math. Model. Numer. Anal., 33 (1999), no. 2, pp. 407-438.

[10] F. KAPPEL AND D. SAlAmon, An approximation theorem for the algebraic Riccati equation, SIAM J. Control Optim., 28 (1990), no. 5, pp. 1136-1147.

[11] V. Komornik, Exact controllability and stabilization, the multiplier method, Wiley, Masson, Paris, 1994.

[12] S. LABBÉ AND E. TRÉLAT, Uniform controllability of semidiscrete approximations of parabolic control systems, Preprint Univ. Paris-Sud, Orsay (2005).

[13] I. LASIECKA, Convergence estimates for semidiscrete approximations of nonselfadjoint parabolic equations, SIAM J. Num. Anal., 21, 5 (1977), pp. 894-908.

[14] I. LASIECKA AND R. TRIGGIANI, Control theory for partial differential equations: continuous and approximation theories. I. Abstract parabolic systems, Encyclopedia of Mathematics and its Applications, 74, Cambridge University Press, Cambridge, 2000. 
[15] G. Lebeau and L. Robbiano, Contrôle exact de l'équation de la chaleur, Comm. Partial Differential Equations, 20 (1995), pp. 335-356.

[16] L. LEOn AND E. ZuAZuA, Boundary controllability of the finite-difference space semi-discretizations of the beam equation, A tribute to J.-L. Lions, ESAIM Control Optim. Calc. Var., 8 (2002), pp. 827-862.

[17] J.-L. Lions, Exact controllability, stabilizability and perturbations for distributed systems, SIAM Rev., 30 (1988), 1-68.

[18] J.-L. LiOns, Contrôlabilité exacte, perturbations et stabilisation de systèmes distribués, Tome 1, Recherches en Mathématiques Appliquées, 8, Masson, 1988.

[19] J.-L. Lions And E. Magenes, Problèmes aux limites non homogènes et applications, Travaux et Recherches Mathématiques, No. 17, 18, 20, Dunod, 1968.

[20] Z. LiU AND S. Zheng, Semigroups associated with dissipative systems, Chapman \& Hall/CRC, Research Notes in Mathematics, 398, 1999.

[21] A. Lopez And E. ZuAzuA, Some new results related to the null controllability of the 1-d heat equation, Séminaire sur les Equations aux Dérivées Partielles, Ecole Polytechnique, VIII (1998), pp. 1-22.

[22] S. Micu, Uniform boundary controllability of a semi-discrete 1-D wave equation, Numer. Math., 91 (2002), no. 4, pp. 723-768.

[23] V. J. Mizel And T. I. Seidman, Observation and prediction for the heat equation, J. Math. Anal. Appl. 28 (1969), 303-312.

[24] M. Negreanu And E. Zuazua, Uniform boundary controllability of a discrete 1-D wave equation, Systems Control Lett., 48 (2003), 3-4, pp. 261279.

[25] A. PAZY, Semigroups of linear operators and applications to partial differential equations, Applied Mathematical Sciences, 44, Springer-Verlag, New York, 1983.

[26] K. Ramdani, T. TAKahashi And M. TUCSnAK, Uniformly exponentially stable approximations for a class of second order evolution equations : application to the optimal control of flexible structures, Preprint Univ. Nancy, 2004.

[27] R. REBARBer AND G. Weiss, Necessary conditions for exact controllability with a finite-dimensional input space, Systems Control Lett., 40 (2000), no. 3, pp. 217-227.

[28] D. L. Russell, Controllability and stabilizability theory for linear partial differential equations: recent progress and open questions, SIAM Rev., 20, 4 (1978), pp. 639-739. 
[29] T. I. Seidman, Observation and prediction for the heat equation, III, J. Differential Equations, 20 (1976), no. 1, 18-27.

[30] L. R. Tcheugoué TéBou and E. Zuazua, Uniform exponential long time decay for the space semi-discretization of a locally damped wave equation via an artificial numerical viscosity, Numer. Math., 95 (2003), no. 3, pp. 563-598.

[31] M. Tucsnak And G. Weiss, Simultaneous exact controllability and some applications, SIAM J. Control Optim., 38 (2000), 5, pp. 1408-1427.

[32] M. TuCSNaK And G. Weiss, How to get a conservative well-posed linear system out of thin air. I. Well-posedness and energy balance, ESAIM Cont. Optim. Calc. Var., 9 (2003), pp. 247-274.

[33] M. Tucsnak and G. Weiss, How to get a conservative well-posed linear system out of thin air. II. Controllability and stability, SIAM J. Control Optim., 42 (2003), 3, pp. 907-935.

[34] G. WeIss, Admissible observation operators for linear semigroups, Israel J. Math., 65 (1989), 1, pp. 17-43.

[35] G. WeIss, Admissibility of unbounded control operators, SIAM J. Control Optim., 27 (1989), 3, pp. 527-545.

[36] E. ZuAZuA, Boundary observability for the finite-difference space semidiscretizations of the 2-D wave equation in the square, J. Math. Pures Appl., 9, 78 (1999), no. 5, pp. 523-563.

[37] E. Zuazua, Controllability of partial differential equations and its semidiscrete approximations, Discrete Contin. Dyn. Syst., 8 (2002), no. 2, pp. 469-513.

[38] E. ZuAzuA, Optimal and approximate control of finite-difference approximation schemes for the 1-D wave equation, Rendiconti di Matematica, SIAM Review, 47, 2 (2005), pp. 197-243.

[39] E. ZuAzuA, Propagation, Observation, Control and Numerical Approximation of Waves approximated by finite difference method, to appear in SIAM Review. 